\title{
Corela
}

Cognition, représentation, langage

11-2| 2013

Vol. $11, \mathrm{n}^{\circ} 2$

\section{Accentuation des composés savants en anglais contemporain : identification morphosémantique et enjeu phonologique}

Fournier Pierre

\section{(2) OpenEdition}

Journals

Édition électronique

URL : http://journals.openedition.org/corela/2971

DOI : $10.4000 /$ corela.2971

ISSN : 1638-573X

Éditeur

Cercle linguistique du Centre et de l'Ouest - CerLICO

Référence électronique

Fournier Pierre, «Accentuation des composés savants en anglais contemporain : identification morphosémantique et enjeu phonologique », Corela [En ligne], 11-2 | 2013, mis en ligne le 07 janvier 2014, consulté le 20 avril 2019. URL : http://journals.openedition.org/corela/2971 ; DOI : 10.4000/ corela.2971

Ce document a été généré automatiquement le 20 avril 2019.

\section{(c) (1) (2)}

Corela - cognition, représentation, langage est mis à disposition selon les termes de la licence Creative Commons Attribution - Pas d'Utilisation Commerciale - Partage dans les Mêmes Conditions 4.0 International. 


\title{
Accentuation des composés savants en anglais contemporain : identification morphosémantique et enjeu phonologique
}

\author{
Fournier Pierre
}

\section{Introduction ${ }^{1}$}

1 L'assignation de l'accent primaire dans les composés savants obéit à une logique spécifique à cette catégorie d'items. Ces considérations phonologiques passent au préalable par un travail de reconnaissance de ces structures en anglais contemporain. Cette identification nécessite une analyse morphosémantique permettant de délimiter les bases classiques. La première étape de l'analyse consiste donc à repérer les différents types de composés savants et à montrer, dans un second temps, l'influence des bases d'origine classique sur le placement de l'accent primaire.

\section{Les composés savants en anglais contemporain}

\subsection{Définition générale}

2 Les dénominations utilisées pour qualifier ce type d'items varient dans la littérature spécialisée. Les auteurs anglo-saxons utilisent généralement l'étiquette «neoclassical compounds» (Plag 2003) ou "Greek-type compounds» (Kingdon 1958). Les morphophonologues français parlent de "constructions savantes» ou de "composés savants ». L'abondance de ces termes en anglais ${ }^{2}$ 'explique en partie par les besoins scientifiques et techniques qui émergent avec l'apparition de nouvelles technologies (Coates 1999: 25). Ce processus de création lexicale mobilise des éléments d'origine 
grecque et latine, avec des possibilités combinatoires importantes en raison du nombre conséquent d'éléments d'origine classique ayant intégré la langue anglaise. La productivité de ces composés en anglais est ainsi extrêmement élevée (Bauer 2001, Trevian 2010). La citation de J.-M. Fournier restitue parfaitement les éléments définitoires des composés savants : «Les composés savants sont formés à partir de bases reprises du grec ou du latin, et essentiellement utilisés dans la terminologie scientifique ${ }^{3}$ : c'est pour ces deux raisons, origine "classique» de la base et utilisation des composés dans le vocabulaire savant, que l'on dénomme ainsi ce groupe particulier de composés " (Fournier $2010: 75$ ).

\subsection{Structure morphologique et statut des éléments}

3 La structure morphologique constitue le facteur principal d'identification des composés savants. Les morphophonologues français utilisent un système de représentation alphabétique qui modélise la structure morphologique (voir Guierre 1979, Duchet 1994, Ginésy 2000). Ainsi, la structure « $A+B+C »$ s'interprète comme un composé savant constitué de deux bases (A et $\mathrm{B}$ ), auxquelles est accolé un suffixe (représenté par la lettre C) ${ }^{4}$. Les bases A et B peuvent être monosyllabiques ou polysyllabiques :

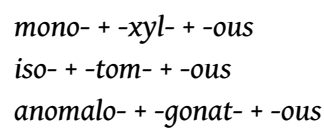

4 S'ajoutent généralement à cette représentation une structure syllabique de la base B en CVC- qui implique la présence d'une voyelle de liaison ${ }^{5}$ et des diacritiques grecs ( $<o e, t h$, $p h, k>$ ) (Trevian 2003). De plus, certaines bases peuvent apparaître en position A ou B, avec les transformations graphiques que cela entraîne (exemple concernant la base -graph - : orthography / graphology).

5 Cependant, le statut des éléments intervenant dans le processus de composition reste flou. La tradition anglo-saxonne, représentée notamment par l'Oxford English Dictionary (OED) et les dictionnaires de prononciation (le Longman Pronunciation Dictionary (LPD) et le Cambridge English Pronouncing Dictionary (EPD)) utilisent le terme « combining form » pour qualifier des séquences telles que -vorous, -phagous ou - philous. La frontière morphologique entre la base B et le suffixe (élément C) n'est donc pas prise en compte, ce qui a une conséquence directe sur la perception du statut de ces séquences. F. Katamba note que ces séquences sont à la frontière des domaines de la composition et de l'affixation (Katamba 1993: 336). Néanmoins, les chercheurs anglo-saxons adoptent majoritairement l'hypothèse de la composition. La tradition morphophonologique française utilise la dénomination "quasi-morphème " ${ }^{6}$, proposée par L. Guierre, pour qualifier le statut des éléments A et B (voir Lilly et Viel 1992, Tournier 1993). La thèse d'état de J. Tournier (1985) considère d'ailleurs l'analyse guierrienne des composés savants comme une avancée sur les ambiguïtés de la notion anglo-saxonne de " combining form ». Le terme de base savante liée, utilisé dans cet article pour qualifier les éléments $\mathrm{A}$ et $\mathrm{B}$, illustre davantage à notre sens l'origine classique et le sémantisme inhérent de ces éléments. Pour représenter le suffixe, la représentation alphabétique $C$ est conservée. Cette représentation des composés savants souligne la présence des frontières morphologiques et symbolise parfaitement le phénomène d'interaction caractérisant ces items. La séparation entre B et $C$ est fondamentale car elle permet grâce au système de commutation d'établir les alternances morphologiques possibles en C. Afin 
d'analyser l'accentuation des composés $A B C$ en anglais, la partie suivante détaille les étapes d'élaboration d'un corpus institutionnalisé représentatif de composés savants en anglais?

\section{Constitution d'un corpus représentatif de composés savants suffixes}

\subsection{Les sources}

6 L'origine de cette recherche remonte à la constitution d'un corpus de données dictionnairiques regroupant tous les suffixés en -ous présents dans l'OED, le LPD et l'EPD (Fournier 2011) ${ }^{8}$. Les composés savants en -ous sont également abordés et de manière très détaillée dans l'ouvrage de I. Trevian (2010: 424-459). Y sont recensés quelques 1500 composés en -ous avec des problématiques morphophonologiques telles que le statut contesté de certaines séquences héritées du latin comme -inous, la variabilité de la voyelle de liaison et les variations de termes engendrées, la question de l'allomorphie éventuelle de certains suffixes (-eous), ou encore les conflits accentuels engendrés par la notion « d'axe dérivationnel ».

7 Cet article ne traite que des composés savants suffixés en -ous attestés dans au moins l'une des trois sources citées ci-dessus. Le LPD et l'EPD sont purement des dictionnaires de prononciation, tandis que l'OED9 est une source dictionnairique étymologique à l'intérieur de laquelle figurent parfois les transcriptions phonétiques associées. La constitution d'un corpus représentatif passe nécessairement par l'application de critères stricts. Les composés savants obsolètes ${ }^{10}$ ne sont pas conservés car ils ne sont pas représentatifs de l'anglais contemporain (exemples: umbriphilous, sexiferous, salutigerous, pterobranchious, prasophagous ${ }^{11}$ ). De plus, les composés dont la transcription phonétique n'est pas attestée ${ }^{12}$ sont écartés car la troisième partie de l'étude intègre une dimension phonologique. Identifier les composés savants suffixés en fonction de critères morphologiques nécessite d'avoir recours à des paramètres sémantiques. En effet, la délimitation morphologique des bases est corrélée à des considérations sémantiques sans lesquelles il est impossible de repérer les frontières. Les contraintes combinatoires régissant la formation de ce type de composés ne sont pas abordées dans le cadre de cette étude car le corpus utilisé est créé à partir de termes attestés en anglais. Une étude sur la productivité de ces composés nécessiterait en revanche une analyse de ces contraintes.

8 Le domaine de spécialisation de l'item constitue également un indice précieux. L'outil étymologique qu'est l'OED permet de classer les composés par domaine sémantique. Cette analyse est exclusivement basée sur ses données ${ }^{13}$. Il permet de distinguer plusieurs types de composés savants.

\subsection{Les différents types de composés savants}

Différentes structures morphologiques sont attestées au sein des composés savants. L'élément $\mathrm{A}$ peut tout d'abord être un préfixe. Le composé savant relève donc de la structure suivante :

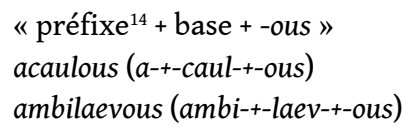




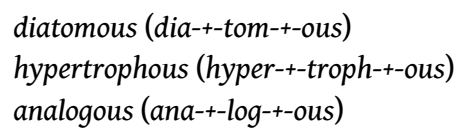

10 Ces composés préfixés ne sont pas classés parmi les composés savants dans les analyses traditionnelles. Or, il s'avère qu'il y a un phénomène de composition entre le préfixe $\mathrm{A}$ et la base $\mathrm{B}$, ainsi qu'un domaine de spécialisation identique à celui des composés avec deux bases. Cette structure regroupe aussi bien des préfixes productifs en anglais contemporain que des préfixes historiques du type apo-, epi-, ou encore cata- aujourd'hui non-productifs.

$11 \mathrm{Au}$ sein des composés savants à deux bases $\mathrm{A}$ et $\mathrm{B}$, plusieurs catégories sont à distinguer. Les composés avec un élément numéral en base A constituent une première souscatégorie. L'étiquette "élément numéral » est employée volontairement car selon les sources étymologiques et morphologiques considérées, il peut s'agir de " préfixes » ou de «bases liées ». Les exemples suivants sont formés sur ce modèle.

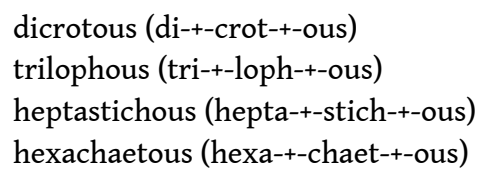

12 La distinction purement sémantique entre référence numérique ou non pose les mêmes problèmes d'identification entre la classe des préfixes et celle des bases liées.

13 Les structures en "base + base + -ous» restantes sont classées en fonction du degré d'attestation de la base $\mathrm{A}$ au sein de l'OED. Il s'avère que certaines des bases sont attestées tandis que d'autres ne le sont pas ${ }^{15}$. En effet, les bases mono-, poly-, homo-, hetero-, iso-, ortho- ou encore phaeno-, sont présentes dans l'OED et ont un potentiel de productivité important au sein de la composition savante (ex: monorhinous, isotomous, polytocous, orthologous, phaenozygous). Cette configuration de constructions savantes est la plus prolifique en anglais. Les bases attestées pour lesquelles une modification ou une élision de la voyelle finale de la base A s'opère sont également associées. Pour illustrer ce phénomène, considérons la base calco-, dont la voyelle finale se modifie lorsqu'elle entre dans un processus compositionnel avec certaines bases B, comme dans calcigerous. Au contraire, les bases *alvi-16, *lipo-, *stamini-, *luci-, *lyo- ou *dolicho- ne sont pas attestées, mais participent tout de même à former des composés savants (ex : alviducous, lipoxenous, luciferous, lyomerous).

14 Le caractère construit des bases $A$ et $B$ représente également un facteur potentiel d'influence sur le placement de l'accent primaire. Il s'avère que des bases telles que nemato-, protero- ou -mycet- sont soit préfixées, soit suffixées. Cette spécificité morphologique place ce type de composés savants en marge des catégories précédentes et modifie leur représentation alphabétique :

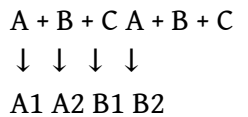

15 Le composé phycomycetous a une base B (-mycet-) elle-même suffixée (B1 -myc-+ B2 -et-) $)^{17}$. La prochaine sous-catégorie de composés savants identifiée est peu prolifique. Elle regroupe tous les termes construits avec trois éléments (à l'exception du suffixe). Ces structures sont représentées de la manière suivante :

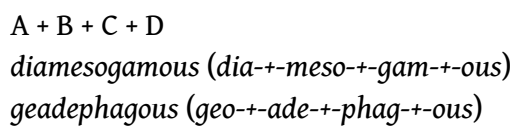


Enfin, une dernière catégorie de composés savants est attestée en anglais. Certaines structures présentent un élément initial séparable (avec un statut de préfixe ou de base) : adiaphanous, adiathermanous, anencephalous, anisogynous, anisomerous, anisopterous, asynchronous, hypodicrotous, androdioecious, andromonoecious, autapomorphous, geosynchronous, gynandromorphous, microlepidopterous, microaerophilous, ovoviviparous, polygynandrous, pseudotetramerous...

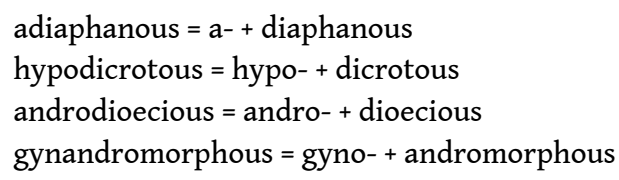

17 L'OED regorge de composés savants présentant cette caractéristique. En conservant le mode de représentation alphabétique, ces structures relèvent de la logique suivante :

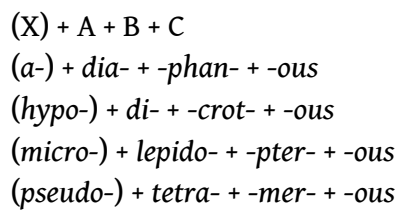

L'élément ( $\mathrm{X}$ ) est séparable car le composé $\mathrm{ABC}$ est attesté dans les sources dictionnairiques. En effet, tous les composés $A B C$ servant de support aux éléments initiaux séparables ci-dessus y sont recensés : diaphanous, diathermanous, encephalous, isogynous, isomerous, isopterous, synchronous, dicrotous, dioecious, monoecious, apomorphous, andromorphous, lepidopterous, aerophilous, viviparous, gynandrous, tetramerous. Il s'agit de l'unique facteur permettant de différencier ces composés avec les composés de type ABCD traités précédemment. En effet, dia-18 dans diamesogamous n'est pas séparable car * mesogamous n'est pas attesté en anglais (le constat est identique pour *adephagous). La distinction entre les deux types de structures a un impact sur leur accentuation. La séparabilité de l'élément initial est généralement sanctionnée d'un comportement accentuel distinct.

Cette classification des composés savants repose essentiellement sur des critères morphologiques, associés à des corrélats sémantiques. La frontière entre la composition savante et le reste du lexique n'est pour autant pas simple à déterminer.

\subsection{Les formes alternatives de composition savante}

20 La catégorie des composés savants avec un élément initial séparable représenté par (X) impose que l'élément $\mathrm{ABC}$ soit lui-même un composé savant. Par conséquent, quel statut accorder aux termes suivants: achylous, anhydrous, antibilious, antistrumous, hypiodous, hypochlorous, hyposulphurous, arteriovenous, chylaqueous, diplarthrous, equisetaceous, heterocystous, hydrocarbonaceous, microvillous, monosiphonous, mucocutaneous, multivarious, myxoedematous, nitrosulphurous, organophosphorous, polysarcous, pyrolignous? Ces structures sont également composées d'un élément initial séparable :

$$
\begin{aligned}
& \text { achylous = a- + chylous } \\
& \text { antistrumous }=\text { anti- }+ \text { strumous } \\
& \text { hypochlorous }=\text { hypo- }+ \text { chlorous } \\
& \text { nitrosulphurous = nitro- + sulphurous }
\end{aligned}
$$

21 Le fait que sulphurous soit attesté n'empêche cependant pas de considérer nitrosulphurous comme un composé savant. En revanche, l'institutionnalisation de BC a un effet sur la position de l'accent $/ 1 /$ des composés $\mathrm{ABC}$ comme nous le verrons dans la troisième 
partie consacrée à l'accentuation de ces structures. Les structures BC ci-dessus ne répondent pas favorablement aux facteurs d'identification de la composition savante. Ces structures, généralement composées d'une seule base suffixée, relèvent du vocabulaire scientifique car le rapport compositionnel inhérent aux constructions savantes n'est pas marqué. Elles ne sont donc pas intégrées au corpus.

Certaines bases $B$ posent également un problème d'ambivalence morphologique entre une utilisation courante et une utilisation savante. Les cas suivants sont particulièrement représentatifs :

apetalous (-petal-)

polypyrenous (-pyren-)

unicolorous (-color-)

En d'autres termes, l'élément mobilisé en B est-il le syntagme nominal attesté dans les sources dictionnairiques (petal, color, pyrene) ou la base savante combinable (-petal-,-color-, -pyren-) ? Le premier critère qui permet de le déterminer est d'ordre sémantique. Le domaine de spécialisation de ces formations étant essentiellement scientifique, l'élément B est employé dans son acception savante. De plus, le second argument corrobore le premier. Plus un élément B est productif dans la formation de composés savants, plus son statut évolue vers celui de base. Ainsi, nous considérons avoir affaire à des composés et non à des constructions de type " $a-+$ petal +-ous». Enfin, certaines formations sont historiquement issues de la composition savante. En revanche, elles ne sont pas conservées en raison d'un usage courant du terme en anglais contemporain. Les termes suivants sont révélateurs puisqu'ils ne se limitent plus à la sphère savante: unanimous, preposterous ou recidivous. La diffusion de ces termes au sein d'une large communauté de locuteurs modifie vraisemblablement les propriétés intrinsèques des bases savantes concernant l'accentuation.

Une identification des composés savants basée sur des critères morphologiques corrélés à des considérations sémantiques est essentielle car les répercussions phonologiques sont importantes, en particulier concernant l'assignation de l'accent primaire ${ }^{19}$ (noté /1/).

\section{Enjeux phonologiques de la classification morphosémantique}

\subsection{Les enjeux de la classification}

L. Guierre remarque dès 1979 que les composés savants présentent des particularités en anglais: "tant sur le plan de leur formation que sur le plan phonétique, les quasimorphèmes ont des propriétés formelles, distributionnelles, accentuelles et segmentales différentes de celles des morphèmes" (Guierre 1979: 737). Le placement de l'accent primaire obéit donc à une logique propre à cette catégorie de composés. La présence du suffixe -ous a un impact fondamental sur la position de l'accent /1/ :

$$
\begin{aligned}
& \text { 'monorhine < mono'rhinous } \\
& \text { 'primigene < pri'migenous } \\
& \text { 'calcifuge < cal'cifugous }
\end{aligned}
$$

L'ajout du suffixe -ous à un dérivant composé de type $\mathrm{AB}$ provoque systématiquement un déplacement de l'accent primaire dans le dérivé. Le suffixe -ous est donc contraignant au sein de la composition savante dans le sens où il interdit la référence au dérivant, caractéristique de la dérivation neutre. Le recours à une analyse dérivationnelle pour 
déterminer le placement de l'accent primaire n'étant donc pas pertinente, l'assignation de l'accent / 1 / est déterminée par un mode de calcul direct. L'élément fondamental dans l'assignation de l'accent /1/ réside dans l'interaction entre la base B et le suffixe (voir Guierre 1979 et Fournier 2011) ${ }^{20}$. La présence d'un dérivant n'étant pas pertinente, le traitement est identique, que les composés suffixés aient un dérivant ou pas. Par conséquent, la typologie des composés savants présentée dans la seconde partie ne prend pas en compte la présence éventuelle d'un dérivant. En revanche, la délimitation de la base B et du suffixe est déterminante car certaines bases B ont un potentiel d'attractivité vis-à-vis de l'accent lorsqu'elles sont suffixées. Cette explication est la seule qui permette d'uniformiser le comportement accentuel des composés suffixés en soulignant un degré de stabilité au niveau de la position de l'accent. Les propriétés phonologiques/ étymologiques de certaines bases $\mathrm{B}^{21}$ sont donc fondamentales dans l'assignation de l'accent principal.

\subsection{Placement de l'accent primaire dans les structures (X)ABC}

Ces formations obéissent à une logique accentuelle différente des autres. La position de l'accent primaire étant déterminée à partir de l'interaction entre la base $\mathrm{B}$ et -ous, l'analyse des composés $(\mathrm{X}) \mathrm{ABC}$ s'avère redondante car les composés savants $\mathrm{ABC}$ sont déjà attestés ${ }^{22}$. La position de l'accent $/ 1 /$ dans les composés (X)ABC est donc identique à celle des composés $\mathrm{ABC}$ correspondants :

andromo'noecious - mo'noecious

equise'taceous - se'taceous

myxoe'dematous - oe'dematous

En revanche, l'assignation de l'accent /1/ dans les autres catégories de composés savants identifiées relève uniquement de l'influence des bases B suffixées vis-à-vis de l'accent /1/.

\subsection{Représentation numérique de l'influence des bases B suffixées sur la position de l'accent primaire}

Comment matérialiser l'influence des bases B suffixées sur le placement de l'accent /1/ ? Le système de représentation utilisé s'inspire du modèle développé par R. Kingdon dans son ouvrage de 1958, en attribuant une représentation numérique symbolisant l'influence de chaque base $\mathrm{B}$ sur le placement de l'accent ${ }^{23}$. Par exemple, l'accent primaire tombe systématiquement sur la syllabe qui précède la base B -phag- dans les 46 cas du corpus :

'

La base -phag- se voit donc attribuer la représentation numérique -1 dans la classification. En revanche, l'accent primaire tombe toujours sur la base -chaet- :

a'chaetous, hexa'chaetous, peri' chaetous, tetra' chaetous

Elle se voit donc attribuer la représentation numérique $+1^{24}$. Le tableau ${ }^{25}$ ci-dessous répertorie le comportement des bases $B$ dont l'influence sur la position de l'accent / 1 / est stable en toute occasion. 
-fer- (249), -phag- (46), -vor- (43), -ger- (36), -phil- (35), -phor- (32), -gam- (27), -pter- (24), -tom(12), -log- (10), -phon- (10), -stom- (9), -gon- (9), -toc- (7), -drom- (5), -phan- (5), -chro- (4), -dom(4), -ton- (6), -clad- (3), -com- (3), -fug- (3), -chthon- (3), -nom- (3), -latr- (3), -bol- (2), -troph- (2), -1 -jug- (2), -xyl- (2), -loq- (2), -bry- (1), -my- (1), -phyt- (1), -petr- (1), -cen- (1), -pil- (1), -pher- (1), -jur- (1), -hum- (1), -dol- (1), -crot- (1), -pno- (1), -steg- (1), -thyr- (1), -dym- (1), -loph- (1), graph- (1), -prag- (1), -der- (1), -phem- (1), -del- (1), -typ- (1), -vom- (1), -cav- (1), -pol- (1), -ten(1), -vag- (1), -men- (1), -lob- (1), -cycl- (1)

-petal- (22), -cephal- (21), -sepal- (9), -dactyl- (8), -onym- (11), -chaet- (6), -chrom- (5), -coel- (5), -dynam- (5), -anim- (5), -stemon- (5), -omal- (3), -thalam- (3), -color- (3), -oic- (3), -caul- (2), -cliv - (2), -anther- (2), -enter- (2), -omphal- (2), -gener- (2), -etr- (2), -stachy- (2), -tech- (2), -agr- (2), -gonat- (2), -(o)ur- (2), -spondyl- (2), -condyl- (1), -l(a)ev- (1), -odyn- (1), -myel- (1), -sbaen- (1), -

+1 metr- (1), -therman- (1), -edr- (1), -tepal- (1), -tuber- (1), -enchym- (1), -brom- (1), -rhiz- (1), hemer- (1), -pneumon- (1), -quetr- (1), -elytr- (1), -pelat- (1), -strat- (1), -om- (1), -a(e)v- (1), -d (a)en- (1), -sug- (1), -ostrac- (1), -gyr- (2), -phaner- (1), -nem- (1), -cerat- (1), -chyl- (1), -cheil(1), -myod- (1), -cotyl- (1), -rhin- (1), -chor- (1), -hedr- (1), -mel- (1), -alter- (1), -crotaph- (1), neur- (1), -mor- (1), -phloe- (1), -poi- (1)

+2 -mycet- (5), -encephal- (3), -pyren- (2), -legomen- (1)

Tableau : Représentation numérique du placement de l'accent / / / dans les composés savants en fonction de l'influence de la base $\mathrm{B}$

La délimitation des bases est opérée à partir de la graphie anglaise, ce qui évite le problème de retranscription des bases vis-à-vis du grec et du latin. Les bases permettant de former un seul composé en -ous sont sujettes à des problèmes de retranscription en raison d'une étymologie parfois incertaine. De la même façon, qualifier de stable leur influence sur la position de l'accent sur la base d'un seul exemple répertorié constitue un paradoxe.

Ce système de représentation numérique présente toutefois l'avantage de mettre en évidence les bases savantes dont l'influence sur le placement de l'accent /1/ est instable.

\begin{tabular}{|l|l|l|l|}
\hline- gen- & $-1(87) ;+1(1)$ & - pyr- & var $-1 /+1(1)$ \\
\hline- par- & $-1(28) ;-2(1) ;-3(1)$ & $-z y g-$ & $-1(1) ;+1(4) ;$ var $-1 /+1(1)$ \\
\hline- col- & $-1(26) ;-2(1)$ & - styl- & $-1(1) ;+1(3)$ \\
\hline- pod- & $-1(24) ;-2(1) ;$ var $-1 /+1(2)$ & - man- & $-1(2) ;$ var -1/+1 (1) \\
\hline- gyn- & $-1(20) ;$ var $-1 /+1(1)$ & - clin- & $-1(1) ;+1(2) ;$ var -1/+1 (1) \\
\hline- mer- & $-1(17) ;+1(1)$ & - spor- & $-1(5) ;+1(3)$ \\
\hline- gnath- & $-1(16) ;$ var $-1 /+1(1)$ & - duc- & $-2(1) ;+1(1)$ \\
\hline- flor- & $+1(13) ;$ var $-1 /+1(1)$ & - phob- & $-1(3) ;+1(2) ;$ var -1/+1 (1) \\
\hline- cer- & $-1(12) ;$ var $-1 /+1(1)$ & - pyg- & $+1(1) ;$ var $-1 /+1(1)$ \\
\hline
\end{tabular}




\begin{tabular}{|l|l|l|l|}
\hline -chron- & $-1(11) ;$ var $-1 /+1(1)$ & -xen- & $-1(1) ;$ var $-1 /+1(1)$ \\
\hline -stomat- & $+1(10) ;$ var $+1 /+2(1)$ & -troch- & $-1(1) ;$ var $-1 /+1(1)$ \\
\hline -stich- & $-1(6) ;$ var -1/+1 (2) & -pach- & var -1/+1 (1) \\
\hline -trop- & $-1(10) ;-2(1) ;$ var -1/+1 (1) & -sem- & $+1(1) ;$ var -1/+1 (1) \\
\hline -glyph- & $-1(1) ;+1(2)$ & -rrhaph- & var -1/+1 (1) \\
\hline -trich- & $-1(5) ;+1(1) ;$ var -1/+1 (2) & - por- & $-1(1) ;+1(2) ;$ var -1/+1 (1) \\
\hline
\end{tabular}

Tableau : Bases savantes B avec une influence instable sur la position de l'accent /1/

Le terme "var » signifie qu'un phénomène de variation accentuelle est attesté, dont les deux variantes sont décrites par les représentations numériques qui suivent. Les composés en -trichous peuvent être accentués de trois façons : cinq items sont accentués sur la syllabe qui précède la base $(-1)$, un seul sur la base $(+1)$ et deux autres attestent un phénomène de variation car ils sont accentués sur la base $\mathrm{B}$ ou bien sur la syllabe précédente $(\mathrm{var}+1 /-1)$.

La base -glyph- génère trois composés en -glyphous dont deux sont accentués sur la base glyph- (+1), le dernier cas étant accentué sur la syllabe précédant la base (-1). L'instabilité de l'influence de la base ne se manifeste pas ici par une variation accentuelle portant sur un seul item, mais pas une variation inter-syntagmatique. L'influence de la base est en effet différente selon les composés.

Les 30 bases ci-dessus n'ont pas une influence stable sur le placement de l'accent primaire. Néanmoins, des disparités apparaissent au sein du tableau. En effet, certaines bases ont une influence globalement stable, à l'exception d'un terme dont la position de l'accent $/ 1 /$ diffère et génère une représentation numérique marginale. L'exemple le plus représentatif est celui de la base -gen-. Dans 87 cas, l'accent primaire tombe sur la syllabe qui précède la base. Le comportement de référence attribué à la base peut donc légitimement être considéré comme -1 . En revanche, la représentation +1 n'est attestée qu'à une seule reprise (bi'genous) dans les composés en -genous. Cette accentuation marginale ne saurait constituer une alternative accentuelle, et ces bases pour lesquelles des comportements accentuels rares sont observés sont donc considérées comme stables. Le tableau ci-dessous répertorie les bases B dont l'influence sur la position de l'accent primaire est stable, tout en indiquant les schémas accentuels marginaux attestés.

\begin{tabular}{|c|c|c|}
\hline bases B & $\begin{array}{l}\text { comportement } \\
\text { de référence }\end{array}$ & comportements rares observés (avec exemples) \\
\hline -gnath- & $-1(16)$ & var $-\mathbf{1} /+\mathbf{1}(\mathbf{1})=$ prog' nathous $^{26}-$ 'prognathous \\
\hline -gen- & $-1(87)$ & $+\mathbf{1}(\mathbf{1})=$ bi'genous \\
\hline -par- & $-1(28)$ & $\begin{array}{l}-2(\mathbf{1})=\text { 'semelparous } \\
-3(\mathbf{1})=\text { 'iteroparous }\end{array}$ \\
\hline
\end{tabular}




\begin{tabular}{|c|c|c|}
\hline -pod- & $-1(24)$ & $\begin{array}{l}-2(1)=\text { 'polypous } \\
\text { var }-1 /+1(2)=\text { myri' apodous - myria' podou } \\
\text { oc'topodous - octo'podous }\end{array}$ \\
\hline -trop- & $-1(10)$ & $\begin{array}{l}-2(1)=\text { 'isotropous } \\
\text { var }-\mathbf{1} /+\mathbf{1}(\mathbf{1})=\text { plagi'otropous - plagio' tropous }\end{array}$ \\
\hline -chron- & $-1(11)$ & var $-1 /+\mathbf{1}(\mathbf{1})=$ me'tachronous - meta' chronous \\
\hline -stomat- & $+1(10)$ & var $+1 /+2(1)=$ mero'stomatous - merosto' matous \\
\hline -gyn- & $-1(20)$ & $\operatorname{var}-1 /+1(\mathbf{1})=$ pseu'dogynous $-p s e u d o ' g y n o u s$ \\
\hline -mer- & $-1(17)$ & $+\mathbf{1}(\mathbf{1})=$ gymno'merous \\
\hline -flor- & $+1(13)$ & $\operatorname{var}-\mathbf{1} /+\mathbf{1}(\mathbf{1})=$ ra' miflorous - rami'florous \\
\hline$-c e r-$ & $-1(12)$ & var $-1 /+1(1)=$ nema'tocerous - nemato'cerous \\
\hline- col- & $-1(26)$ & $-2(\mathbf{1})=m e^{\prime}$ tallicolous \\
\hline
\end{tabular}

Tableau 3 : Comportements de référence des bases B accompagnés des cas marginaux

39 Le comportement de référence est déterminé à partir du nombre de composés relevant des schémas accentuels. La limite permettant de considérer que l'influence d'une base est stable et que les autres schémas accentuels sont marginaux est arbitrairement fixée à dix items. Ainsi, les bases B réellement instables vis-à-vis de la position de l'accent primaire sont répertoriées en annexe, accompagnées des exemples correspondants. La productivité est donc un paramètre déterminant dans l'attribution d'un comportement à une base. Plus une base est productive, et plus l'influence qu'elle a sur le placement de l'accent primaire est stable. En revanche, les bases peu attestées génèrent davantage de schémas accentuels différents (voir les disparités en annexe).

L'influence des bases B intervenant dans la composition savante étant déterminée, il convient de replacer l'accentuation de cette catégorie spécifique d'items dans une perspective plus large. La prochaine sous-partie tente de rendre compte de l'accentuation des composés savants en anglais avec un minimum de règles.

\subsection{Uniformisation de l'accentuation des composés savants}

41 Comment uniformiser le comportement accentuel des composés savants en -ous? Observons tout d'abord la position de l'accent /1/ dans l'ensemble du corpus. 


\begin{tabular}{|l|l|l|l|l|}
\hline$/(-) \mathbf{1 0}$ & $/(-) \mathbf{1 0} /-/(-) \mathbf{1 0 0} /$ & $/(-) \mathbf{1 0 0} /$ & autres & total \\
\hline 103 & 25 & 1053 & 6 & 1187 \\
\hline $8,7 \%$ & $2,1 \%$ & $88,7 \%$ & $0,5 \%$ & \\
\hline
\end{tabular}

Tableau 4 : Placement de l'accent / 1/ dans les composés savants en -ous (-)10/ preuve de l'influence de la base sur l'accentuation. La règle d'accentuation est la suivante :

base savante attractive + -ous $\rightarrow(/(-) 10 /)$ 

primaire passe par un travail préliminaire d'identification. Cette reconnaissance est de nature morphologique et elle est associée à des corrélats sémantiques. Seule l'interpénétration des strates morphologique et phonologique permet de mettre en évidence la spécificité de ces bases d'origine classique sur l'accentuation. Déterminer le comportement accentuel des composés savants sur le concept d'attractivité accentuelle permet de rendre compte de la quasi-totalité des accentuations. La prévisibilité du placement accentuel semble être améliorée si le rôle joué par la base B est spécifié. Cette classification opérée sur un corpus de composés en -ous doit cependant être testée sur d'autres suffixes, et notamment le suffixe -al qui entretient de nombreuses similitudes avec -ous, afin de déterminer si l'influence des bases B est identique quel que soit le suffixe considéré. 


\section{BIBLIOGRAPHIE}

Amiot, D. \& G. Dal (2008), « La composition néoclassique en français et ordre des constituants », La composition dans les langues (D. Amiot éd.), 89-113, Arras : Artois Presses Université.

Bauer, L. (2000), English Word-Formation, Cambridge, New York, Melbourne : Cambridge University Press.

Bauer, L. (2001), Morphological Productivity, Cambridge : Cambridge University Press.

Cellard, J. (1998), Les racines grecques du vocabulaire français, Paris, Bruxelles : Duculot.

Chomsky, N. \& M. Halle (1968), The Sound Pattern of English, Cambridge, Massachusetts, London, England : The MIT Press.

Coates, R. (1999), Word Structure, New York : Routledge.

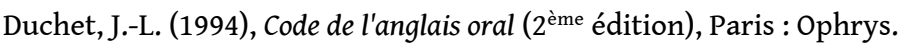

Fournier, J.-M. (2007), « From a Latin syllable-driven stress system to a Romance versus Germanic morphology-driven dynamics : in honour of Lionel Guierre », Language Sciences, 29(2), 218-236.

Fournier, J.-M. (2010), Manuel d'anglais oral, Paris : Ophrys.

Fournier, P. (2011), Accentuation et prononciation des suffixés en -ous en anglais contemporain, Thèse de doctorat, Université de Tours.

Fradin, B. (2003), Nouvelles approches en morphologie, Paris : Presses Universitaires de France.

Guierre, L. (1979), Essai sur l'accentuation en anglais contemporain - Eléments pour une synthèse, Thèse de Doctorat, Université Paris VII.

Guyot-Talbot, A. (2011), « Les frontières de la préfixation : problèmes d'identification et de recoupements. Préfixes et quasi-léxèmes ", Actes du 12ème Colloque d'avril sur l'anglais oral (Nicaise, A. \& R. Huart éds.), 71-92, Université de Paris-Nord : CELDA, diffusion APLV.

Jones, D. (2008), Cambridge English Pronouncing Dictionary (17 ${ }^{\text {th }}$ edition), Cambridge : Cambridge University Press (revised by Roach P., Hartman J. \& J. Setter).

Katamba, F. (1993), Morphology, New York : Palgrave.

Kingdon, R. (1958), The Groundwork of English Stress, London : Longmans, Green.

Lilly, R. \& M. Viel (1999), Initiation raisonnée à la phonétique de l'anglais, Paris : Hachette Université.

Namer, F. \& R. Baud (2007), "Defining and Relating Biomedical Terms: Towards a Cross-language Morphosemantics-based System”, International Journal of Medical Informatics 76, 226-233.

Paillard, M. (2000), Lexicologie contrastive anglais - français, Paris : Ophrys.

Simpson, J. \& E. Weiner (1989), Oxford English Dictionary. Online Edition, Oxford : Clarendon Press.

Tournier, J. (1985), Introduction descriptive à la lexicogénétique de l'anglais contemporain, Genève : Champion Slatkine.

Tournier, J. (1993), Précis de lexicologie anglaise (3 ${ }^{\mathrm{ème}}$ édition), Paris : Nathan.

Trevian, I. (2003), Morphoaccentologie et processus d'affixation de l'anglais, Bern : Peter Lang. 
Trevian, I. (2010), Les affixes anglais, productivité, formation de néologismes et contraintes combinatoires. De la diachronie à la synchronie, Berne : Peter Lang.

Wells, J.C. (2008), Longman Pronunciation Dictionary (3 ${ }^{\text {rd }}$ edition), London : Longman.

\section{ANNEXES}

Annexe : Bases savantes B avec une influence instable sur le placement de l'accent primaire

\begin{tabular}{|c|c|c|}
\hline bases & $\begin{array}{l}\text { comportements } \\
\text { observables }\end{array}$ & exemples correspondants \\
\hline -stich- & $\begin{array}{l}-1(6) \\
\operatorname{var}-1 /+1(2)\end{array}$ & $\begin{array}{l}\text { 'distichous / hep'tastichous / oc'tastichous / pen'tastichous... } \\
\text { mo'nostichous - mono'stichous / or'thostichous - ortho'stichous }\end{array}$ \\
\hline -trich- & $\begin{array}{l}-1(5) \\
+1(1) \\
\operatorname{var}-1 /+1(2)\end{array}$ & $\begin{array}{l}\text { ho'lotrichous / cy'motrichous / lis'sotrichous / u'lotrichous... } \\
\text { amphi'trichous } \\
\text { mo'notrichous - mono'trichous / pe'ritrichous - peri'trichous }\end{array}$ \\
\hline -glyph- & $\begin{array}{l}-1(1) \\
+1(2)\end{array}$ & $\begin{array}{l}\text { 'aglyphous } \\
\text { opistho'glyphous / protero'glyphous }\end{array}$ \\
\hline$-p y r-$ & var $-1 /+1(1)$ & a'pyrous /'apyrous \\
\hline$-z y g-$ & $\begin{array}{l}+1(4) \\
-1(1) \\
\operatorname{var}-1 /+1(1)\end{array}$ & $\begin{array}{l}\text { di'zygous / hemi'zygous / mono'zygous / phaeno'zygous } \\
\text { apha'nozygous } \\
\text { 'azygous - a'zygous }\end{array}$ \\
\hline -styl- & $\begin{array}{l}-1(1) \\
+1(3)\end{array}$ & $\begin{array}{l}\text { 'systylous } \\
\text { di'stylous / meso'stylous / micro'stylous }\end{array}$ \\
\hline -man- & $\begin{array}{l}-1(2) \\
\operatorname{var}-1 /+1(1)\end{array}$ & $\begin{array}{l}\text { 'bimanous / lon'gimanous } \\
\text { qua'drumanous - quadru'manous }\end{array}$ \\
\hline -clin- & $\begin{array}{l}+1(2) \\
-1(1) \\
\operatorname{var}-1 /+1(1)\end{array}$ & $\begin{array}{l}\text { patro'clinous / mono'clinous } \\
\text { 'diclinous } \\
\text { pa'triclinous - patri'clinous }\end{array}$ \\
\hline -spor- & $\begin{array}{l}-1(5) \\
+1(3)\end{array}$ & $\begin{array}{l}\text { a'crosporous / angi'osporous / hete'rosporous / ho'mosporous / zo'osporous } \\
\text { mono'sporous / poly'sporous / tri'sporous }\end{array}$ \\
\hline$-d u c-$ & $\begin{array}{l}+1(1) \\
-2(1)\end{array}$ & $\begin{array}{l}\text { alvi'ducous } \\
\text { 'felli,ducous }{ }^{29}\end{array}$ \\
\hline
\end{tabular}




\begin{tabular}{|c|c|c|}
\hline -por- & $\begin{array}{l}+1(1) \\
-1(1) \\
\operatorname{var}-1 /+1(1)\end{array}$ & $\begin{array}{l}\text { micro'porous, stromato'porous } \\
\text { po'lyporous } \\
\text { ma'croporous / macro'porous }\end{array}$ \\
\hline -phob- & $\begin{array}{l}-1(3) \\
+1(2) \\
\operatorname{var}-1 /+1(1)\end{array}$ & $\begin{array}{l}\text { cal'ciphobous / hae'mophobous / hy'drophobous } \\
\text { ombro'phobous / photo'phobous } \\
\text { ni'trophobous / nitro'phobous }\end{array}$ \\
\hline -pyg- & $\begin{array}{l}+1(1) \\
\operatorname{var}-1 /+1(1)\end{array}$ & $\begin{array}{l}\text { calli'pygous } \\
\text { stea'topygous / steato'pygous }\end{array}$ \\
\hline -xen- & $\begin{array}{l}-1(1) \\
\operatorname{var}-1 /+1(1)\end{array}$ & $\begin{array}{l}\text { li'poxenous } \\
\text { mo'noxenous / mono'xenous }\end{array}$ \\
\hline -troch- & $\begin{array}{l}-1(1) \\
\operatorname{var}-1 /+1(1)\end{array}$ & $\begin{array}{l}\text { ho'lotrochous } \\
\text { po'lytrochous / poly'trochous }\end{array}$ \\
\hline -pach- & $\operatorname{var}-1 /+1(1)$ & i'sopachous / iso'pachous \\
\hline -sem- & $\begin{array}{l}+1(1) \\
\operatorname{var}-1 /+1(1)\end{array}$ & $\begin{array}{l}\text { mono'semous } \\
\text { po'lysemous / poly'semous }\end{array}$ \\
\hline -rrhaph- & $\operatorname{var}-1 /+1(1)$ & or' thorrhaphous / orthor'rhaphous \\
\hline
\end{tabular}

\section{NOTES}

1. Je tiens à remercier chaleureusement les deux relecteurs anonymes de la revue pour leurs commentaires pertinents concernant l'étude et l'élaboration de l'article.

2. Le processus de création des composés savants est identique en français. Ainsi, le raisonnement sur la structure des composés savants anglais vaut également pour le français.

3. Notamment dans le domaine biomédical (Namer et Baud 2007).

4. Certains composés savants de type $\mathrm{ABC}$ admettent des dérivants de type $\mathrm{AB}$ qui obéissent à des logiques accentuelles différentes de leurs dérivés. L'accent primaire tombe en général sur la première ou la seconde syllabe de $\mathrm{A}$. Les composés $\mathrm{AB}$ ne sont pas traités dans le cadre de cette étude. La représentation $A B C$ ne place pas le suffixe sur le même plan morphologique que les bases $A$ et $B$, mais son indéniable influence sur le placement de l'accent $/ 1 /$ dans son interaction avec la base $B$, justifie une analyse distincte des composés $A B$ et l'attribution d'un statut morphophonologique au suffixe avec la représentation $C$.

5. La présence d'un $<0>$ souligne traditionnellement l'origine grecque de la base tandis que le $<\mathrm{i}>$ renvoie à une origine latine.

6. M. Paillard propose le terme de « fracto-morphème » (Paillard 2000).

7. La composition savante en français obéit aux mêmes règles de construction morphologique (Amiot et Dal 2008).

8. Ce choix méthodologique suscite plusieurs questions. Comment les locuteurs prennent-ils connaissance des composés savants? Ces termes, généralement peu productifs lors des interactions orales, sont-ils appréhendés par les locuteurs par l'intermédiaire de l'oral ou de 
l'écrit? Ces questions sont essentielles car l'attribution d'un schéma accentuel dépend également du degré de familiarité que le locuteur entretient avec le domaine. En d'autres termes, il est fort probable qu'une biologiste accentuera correctement un composé savant relatif à son domaine en raison de sa connaissance de la structure morphologique du composé, tandis qu'un locuteur «lambda» confronté à des bases peu transparentes, voires opaques, accentuera vraisemblablement le terme indépendamment de sa structure morphologique. Une étude confrontant les données dictionnairiques à des données orales enregistrées auprès de locuteurs natifs serait particulièrement intéressante dans cette optique, de même que l'étude des conditions d'apparition et d'accentuation de ces composés dans les dictionnaires. Notre corpus se limite ainsi aux formes institutionnalisées présentes dans les trois sources sans s'interroger sur la pertinence des schémas accentuels proposés.

9. Travailler avec l'édition en ligne de l'OED donne accès aux variantes (exemple : mesoconchous / mesokonchous). Toutefois, ces variations ne semblent pas modifier les propriétés des bases B vis-àvis de l'accentuation. Par conséquent, seuls les composés figurant en entrée principale sont conservés.

10. L'OED constitue l'unique source pour déterminer le caractère obsolète ou non du composé. Le LPD et l'EPD ne répertorient quant à eux que des items attestés en anglais contemporain.

11. Voir la version en ligne de l'OED pour plus de détails concernant l'étymologie et la définition de ces items.

12. Lorsque ce cas de figure se présente, le composé savant n'est attesté que par l'OED qui ne lui attribue pas de transcription phonétique. Si le composé devait être attesté dans le LPD et/ou l'EPD, il se verrait nécessairement adjoindre une transcription.

13. La liste des bases A et B productives au sein de la composition savante en -ous, accompagnée des données étymologiques et sémantiques, ainsi que tous les composés savants constituant ce corpus sont disponibles en annexes de notre travail de doctorat (Fournier 2011: 506-536). Ce travail est accessible en ligne à partir du site www.theses.fr.

14. La distinction entre le statut de base et celui de préfixe est opérée à partir des données de l'OED. Le choix de poser l'OED en arbitre des phénomènes morphologiques est purement arbitraire. En effet, le statut de certains éléments ne fait pas consensus au sein de la communauté scientifique (ex : hypo- ou mono-). La distinction entre préfixe et base est donc sujette à caution. Toutefois, afin de ne pas multiplier les différentes dénominations, cette étude adopte le seul point de vue de l'OED. Ainsi, ambi- est davantage considéré comme une « combining form » dans le reste de la littérature spécialisée.

15. Ou tout du moins ne le sont pas encore à l'heure actuelle. Il est fort probable que l'inventaire des «combining forms" présentes dans l'OED ne soit pas identique dans d'autres sources. Certaines sont en passe d'intégrer les zones stables du lexique tandis que d'autres ont encore un potentiel compositionnel moindre.

16. Le symbole * indique que la base n'est pas attestée dans les données de l'OED.

17. Les coupures morphologiques sont effectuées à partir de la forme graphique du composé, telle qu'elle existe en anglais contemporain, et ne prennent pas en compte les spécificités des graphies grecques ou latines.

18. Qu'il s'agisse d'un réel préfixe ou non.

19. Seule la variété accentuelle britannique est extraite des bases de données dictionnairiques. Le degré variable de familiarité avec le terme scientifique peut expliquer une partie des variantes recensées dans l'OED, et cette propriété vaut sans doute pour l'ensemble des locuteurs, toutes variétés confondues.

20. L'élément A ne représente pas un facteur déterminant dans l'assignation de l'accent $/ 1 /$. Le statut de l'élément A est en effet beaucoup trop aléatoire (préfixe productif, préfixe historique, base attestée ou non) pour qu'il joue un rôle significatif dans le placement de l'accent. 
21. Pour le rôle des bases latines en anglais et l'impact de l'introduction du latin sur le système phonologique anglais, voir l'article de J.-M. Fournier (2007).

22. Voir A. Guyot-Talbot (2011) pour plus de renseignements sur le statut et l'accentuation de ces composés avec une partie initiale séparable.

23. Notre méthode s'inspire du système de représentation numérique de R. Kingdon mais récuse toutefois son hypothèse qui assigne l'accent primaire en fonction d'une typologie des «bases liées » de type -vorous ou -philous.

24. La représentation +2 signifie par conséquent que l'accent $/ 1 /$ tombe sur la seconde syllabe de la base B. Les bases B avec une représentation +2 sont donc polysyllabiques.

25. Le chiffre entre parenthèses indique le nombre de composés du corpus construits à partir de la base B associée. L'ordre d'apparition des bases B dans chaque cellule du tableau est dicté par la fréquence.

26. La base B -gnath- pose des problèmes de resyllabification en raison de contraintes phonotactiques. La consonne / $\mathrm{g} /$ relève de la coda de la base A et non de la base liée B. Il s'agit donc d'une base liée hétérosyllabique sur le plan phonologique. Ceci explique la position de l'accent primaire dans prognathous.

27. Les bases semi-attractives sont également incorporées dans la démonstration car leur statut intermédiaire est forcément lié à un comportement particulier de la base.

28. Les structures héritées du grec correspondent pour la plupart à des formes grecques latinisées passées par le système latin.

29. Le schéma en /1020/ de felliducous pose question. Deux interprétations s'opposent: soit l'accent /1/ est effectivement placé sur la première syllabe et auquel cas la base -duc- se voit attribuer le comportement (-2), soit nous intervenons de façon subjective sur les données accentuelles en proposant en schéma en /2010/, conforme à l'accentuation du second adjectif construit à partir de la base -duc-. La première option est retenue puisque rien ne nous autorise à modifier les données de l'OED.

\section{RÉSUMÉS}

L'accentuation des composés savants en anglais contemporain pose un certain nombre de problèmes théoriques. Le critère morphologique est fondamental dans la reconnaissance de ces structures, puisqu'elles obéissent à une interaction de type compositionnel entre des bases et des affixes. L'analyse morphologique est corrélée à des paramètres sémantiques nécessaires à l'identification des bases d'origine classique. Les frontières de la composition savante demeurent néanmoins floues. Le facteur morphosémantique s'avère indispensable dans la détermination du placement de l'accent primaire. L'analyse d'un corpus de composés savants suffixés en -ous, constitué à partir de données dictionnairiques, permet un traitement quantitatif du phénomène. L'observation des schémas accentuels accrédite l'hypothèse selon laquelle la relation entre les bases précédant -ous et ce même suffixe est déterminante dans l'assignation de l'accent. Grâce à un système de représentation numérique modélisant l'influence des bases sur l'accentuation, certaines bases sont qualifiées d'attractives car elles retiennent l'accent primaire tandis que la majorité d'entre elles est non-attractive car ces dernières ne s'opposent pas à la remontée de l'accent sur la syllabe antépénultième. 
Stress assignment in learned compounds in contemporary English raises a number of theoretical problems. The morphological criterion is essential to identify these structures for they result from an interaction between several elements (classical roots and affixes). This morphological analysis is carried along with a semantic perspective which is necessary to determine the roots and to identify the scientific domains. It is difficult to determine the exact limits of learned compounds. Nevertheless, this morphosemantic study turns out to be essential in the assignment of primary stress. Thus, the analysis of a dictionary-based corpus composed of learned compounds suffixed in -ous, enables a quantitative study. An overview of stress patterns gives credit to the hypothesis that there is an interaction between the classical roots preceding the suffix, and this particular suffix, which is the crucial factor in word stress assignment. When using a numerical system of representation which models the influence of roots relative to stress location, some roots appear to be "attractive" because they carry primary stress, while the majority of them are "non-attractive" for they do not prevent primary stress retraction on the antepenultimate syllable.

INDEX

Mots-clés : composition savante, structure morphologique, phonologie, accent primaire

Keywords : learned compounds, morphological structure, phonology, primary stress

\section{AUTEUR}

FOURNIER PIERRE

CRIDAF EA0453, Université Paris 13 - Sorbonne Paris Cité 